\title{
Emerging role of FGF receptors in papillomavirus replication
}

\author{
Leny Jose ${ }^{1}$, Elliot J Androphy ${ }^{1,2}$ \& Marsha DeSmet ${ }^{*, 1}$ \\ ${ }^{1}$ Department of Dermatology, Indiana University School of Medicine, Indianapolis, IN 46202, USA \\ ${ }^{2}$ Department of Microbiology \& Immunology, Indiana University School of Medicine, Indianapolis, IN 46202, USA \\ *Author for correspondence: mdesmet@iu.edu
}

\begin{abstract}
"We envision that FGFR phosphorylates E2 during early stages of viral replication and maintenance to restrict E2 activity from over-replication to prevent lytic infection."
\end{abstract}

First draft submitted: 7 August 2018; Accepted for publication: 6 September 2018; Published online: 19 October 2018

Keywords: E2 • FGFR • HPV • tyrosine phosphorylation $\bullet$ viral replication

Human papillomaviruses (HPV) are small DNA tumor viruses infecting cervix, mouth and throat tissues leading to cervical, oropharyngeal and anogenital cancers [1]. The HPV being an intra-epithelial pathogen, the long-term survival and replication of the virus inside the cell necessitates a two-pronged approach. First, the virus should evade the host immune response. Second, the epithelia are subject to growth factor-dependent proliferation and differentiation programming where the virus has to adapt to the dynamic cellular microenvironment. The replicative lifecycle of the virus is linked to the epithelial differentiation program $[2,3]$. The initial infection occurs in the basal cells in which the viral genome replicates but persists as low copy-number episomes. During this stage, the virus must undergo replication but not over-replicate as to not permit lytic infection. Next, the viral genome is maintained as a stable multicopy episome in dividing basal and suprabasal cells in a cell cycle dependent manner. During this maintenance mode, it is assumed that the viral genomes replicate once per cell cycle during S-phase of cellular division. Monolayer cultures harboring viral episomes mimic this stage.

The E2 and E1 proteins are the initially expressed viral proteins encoded by all HPVs and it is believed that basal levels of E2 are required for the initiation of viral replication and transcription. Structurally, E2 consists of an Nterminal transactivation domain (TAD), a nonconserved hinge region and a DNA-binding domain. Functionally, E2 is a sequence-specific DNA-binding protein, binding to E2- binding sites ACCGN 4 CGGT in the upstream regulatory region and recruiting the viral E1 helicase [4]. The E2 activates transcription of the early promoter and tethers viral genomes to host mitotic chromosomes in basal cells. As infected cells migrate into the upper epithelial strata, the differentiation program is triggered, viral genomes are amplified to hundreds of copies and packaged within the nucleus into virions requiring robust $\mathrm{E} 2$ activity.

The molecular mechanisms by which the viral genome switches from the initial latent maintenance mode of replication to amplification mode during epithelial differentiation is unclear. An increased E2 expression may explain this phenomenon. However, we and others have identified E2 post-translational modifications that are regulatory in nature [5-8]. Among these PTMs, tyrosine phosphorylations are of particular interest since our data suggest that these contribute to switching mechanism from once per cell cycle genome replication to genome amplification.

We recently discovered a phosphorylation of BPV E2 at tyrosine 102 in the TAD. The phosphomimetic tyrosineto-glutamate E2 mutation was unable to bind to the viral helicase E1 and the transcription activator protein Brd4 and was defective for replication and transcription [6]. Growth factor receptors play a major role in cellular reprogramming during viral infection hence, we hypothesized that any of these receptor tyrosine kinases may be responsible for Y102 phosphorylation. Paolini et al. observed an interaction between HPV-16 E2 and the ubiquitin ligase Nrdp-1, which is necessary for regulating the ErbB-3 (HER-3), EGFR family and viral integration [9]. We did not observe an association between E2 and the EGFR or the EphB2, but discovered that FGFRs modulate E2 activity $[10,11]$. 
The FGFRs (1-4) are a group of transmembrane tyrosine kinase receptors with multiple isoforms [12]. The FGFRs possess three extracellular immunoglobulin-like domains, a hydrophobic transmembrane domain and a cytoplasmic kinase domain [13]. They are activated by FGF which binds with high affinity to the FGFRs [14]. The FGF-signaling pathway regulates many biological pathways like proliferation, differentiation, angiogenesis and cell survival. Heparan sulfate proteoglycans modulate the binding of FGFRs to FGFs, inducing heterodimerization and subsequent activation and phosphorylation of FGFRs [15]. Interestingly, cell surface heparan sulfate is essential for successful HPV infection [16,17]. Specifically syndecan-1, a heparan sulfate proteoglycan, was shown to be a cellular receptor for HPV [18]. The heparan sulfate-decorated virus physically interacts with EGFR and KGFR or FGFRllb [19]. However, the interactions of the virus with other growth factor receptors are not known and it is possible that HPV entry may be associated with FGFR activation.

Protein expression signatures from Human Protein Atlas database $[20,21]$ revealed that FGFR1-4 are expressed in keratinocytes; FGFR2 and FGFR3 are abundantly expressed while FGFR1 and FGFR4 are expressed at lower levels [10]. Subsequently, we found that FGFR2 and FGFR3 were in complex with PV E2 proteins [10,11]. Interestingly, FGFR2 and FGFR3 but not FGFR1 and FGFR4 are strongly bound to BPV E2 protein. In contrast, all the four FGFR kinases coimmunoprecipitated with the high-risk HPV-16 and -31 E2 proteins suggesting that the interaction may be genotype and tissue specific. It should be noted that the interaction took place between nuclear FGFR and E2, so nuclear localization of the receptors is evident. We found that the FGFR induced E2 tyrosine phosphorylation. We next ventured to address the viral genome replication in an activated FGFR context in keratinocytes during the maintenance phase of the viral lifecycle. In a transient transfection model, FGFR2 and FGFR3 activation both significantly impaired E2-mediated PV origin replication. The FGFR3 depletion using RNAi significantly enhanced copy number in cells maintaining PV episomes while depletion of another related growth factor tyrosine kinase receptor, EGFR had no effect on viral replication. Although, FGFR activation has the same phenotype of PV replication as the phosphomimetic Y102 (Y102E) E2 mutant, we found no evidence for the target kinase of Y102 being FGFR2 or -3, leading us to believe there is another kinase responsible for Y102 phosphorylation and different tyrosines within E2 are phosphorylated by FGFR.

Despite our understanding of the inhibitory role of FGFR-induced E2 phosphorylation, the exact mechanism of impaired viral replication remains unclear. Phosphorylation of E2 at multiple tyrosines remains a strong probability when the viral replication needs to be limited. Mass spectrometry approaches were used to identify tyrosine phosphorylations of E2 after FGFR2 activation. We observed several tyrosine phosphorylations mostly in the TAD (tyrosines: 32, 44, 131, 158, 159, 169 and 170) of BPV-1 E2 suggesting that protein-protein interactions may be influenced by FGFR-induced E2 phosphorylation [11]. For example, Y32 directly faces E1 and Y44 is oriented towards the Brd4 c-terminus motif and phosphorylation could disrupt the E2 binding to these proteins. The Y131 has been shown to be crucial for Chlr1 binding to E2, which is necessary for viral genome segregation and genome maintenance [22]. We speculate that some of these phosphorylations are FGFR targets and may be the key to understanding the inhibitory function of $\mathrm{E} 2$.

The dephosphorylating mechanisms may come into play during epithelial differentiation and can involve tyrosine phosphatases directly acting on E2 or FGFRs. We envision that FGFR phosphorylates E2 during early stages of viral replication and maintenance to restrict E2 activity from over-replication to prevent lytic infection. During differentiation, FGFR activity or expression is restricted so that the inhibitory phosphorylation of E2 is removed to enhance E2 activity and genome amplification. The potential partnership between FGFR and E2 may explain the molecular switch between viral maintenance and viral amplification.

The FGFR inhibitors are currently in preclinical and clinical development [23-25]. An inhibition of FGFRs in basal cells maintaining episomal HPV can lead to excessive E2-mediated viral replication. We propose two fates for the virus in this scenario. An immune clearance of the virus is a potential outcome as the higher viral load may lead to lytic infection in the proliferating cells and may alert host defense mechanisms. Alternatively, over-replication may lead to viral genome integration and prevent further dissemination of virus to the upper strata of differentiating epithelia. In our viewpoint, the consequence of inhibition of FGFR-E2 interaction needs to be studied further in context of the virus lifecycle. These studies may include E2 mutations at tyrosine(s) which are direct targets of FGFR. Nonetheless, the growth factor-dependent repressive mechanism used by HPV to survive and replicate inside the host cells need to be studied in detail. 


\section{Financial \& competing interests disclosure}

Research discussed in this publication was supported by the National Cancer Institute and National Institute of Allergy and Infectious Diseases of the NIH: R01CA058376 (EJA) and T32AI060519 (MD). The content is solely the responsibility of the authors and does not necessarily represent the official views of the $\mathrm{NIH}$. The authors have no other relevant affiliations or financial involvement with any organization or entity with a financial interest in or financial conflict with the subject matter or materials discussed in the manuscript apart from those disclosed.

No writing assistance was utilized in the production of this manuscript.

\section{References}

1. Serrano B, Brotons M, Bosch FX, Bruni L. Epidemiology and burden of HPV-related disease. Best Pract. Res. Clin. Obstet. Gynaecol. 47, 14-26 (2018).

2. Hong S, Laimins LA. Regulation of the life cycle of HPVs by differentiation and the DNA damage response. Future Microbiol. 8, 1547-1557 (2013).

3. McKinney C, Hussmann K, McBride A. The role of the DNA damage response throughout the papillomavirus life cycle. Viruses 7(5), 2450 (2015).

4. Androphy EJ, Lowy DR, Schiller JT. Bovine papillomavirus E2 transactivating gene product binds to specific sites in papillomavirus DNA. Nature 325, 70 (1987).

5. Chang S-W, Liu W-C, Liao K-Y, Tsao Y-P, Hsu P-H, Chen S-L. Phosphorylation of HPV-16 E2 at serine 243 enables binding to Brd4 and mitotic hromosomes. PLoS ONE 9(10), e110882 (2014).

6. Culleton SP, Kanginakudru S, DeSmet M et al. Phosphorylation of the bovine papillomavirus E2 protein on tyrosine regulates its transcription and replication functions. J. Virol. 91(2), e01854-e01816 (2017).

7. Quinlan EJ, Culleton SP, Wu S-Y, Chiang C-M, Androphy EJ. Acetylation of conserved lysines in bovine papillomavirus E2 by p300. J. Virol. 87(3), 1497-1507 (2013).

8. Thomas Y, Androphy EJ. HPV replication regulation by acetylation of a conserved lysine in the E2 protein. J. Virol. 92(3), pii: e01912-17 (2018)

9. Paolini F, Curzio G, Melucci E et al. Human papillomavirus 16 E2 interacts with neuregulin receptor degradation protein 1 affecting ErbB-3 expression in vitro and in clinical samples of cervical lesions. Eur. J. Cancer 58, $52-61$ (2016).

10. Xie F, DeSmet M, Kanginakudru $S$ et al. Kinase activity fibroblast growth factor receptor-3 regulates activity of the papillomavirus E2 protein. J. Virol. 91(20), pii: e01066-17 (2017).

11. DeSmet M, Kanginakudru S, Jose L, Xie F, Gilson T, Androphy EJ. Papillomavirus E2 protein is regulated by specific fibroblast growth factor receptors. Virology 521, 62-68 (2018).

12. Gong SG. Isoforms of receptors of fibroblast growth factors. J. Cell. Physiol. Suppl. 229(12), 1887-1895 (2014).

13. Gallo LH, Nelson KN, Meyer AN, Donoghue DJ. Functions of fibroblast growth factor receptors in cancer defined by novel translocations and mutations. Cytokine Growth Factor Rev. 26(4), 425-449 (2015).

14. Zhang X, Ibrahimi OA, Olsen SK, Umemori H, Mohammadi M, Ornitz DM. Receptor specificity of the fibroblast growth factor family: the complete mammalian FGF Family. J. Biol. Chem. 281(23), 15694-15700 (2006).

15. Mohammadi M, Olsen SK, Ibrahimi OA. Structural basis for fibroblast growth factor receptor activation. Cytokine Growth Factor Rev. 16(2), 107-137 (2005).

16. Giroglou T, Florin L, Schäfer F, Streeck RE, Sapp M. Human papillomavirus infection requires cell surface heparan sulfate. J. Virol. 75(3), 1565-1570 (2001).

17. Kumar A, Jacob T, Abban CY, Meneses PI. Intermediate heparan sulfate binding during HPV 16 infection in HaCaTs. Am. J. Ther. 21(5), 331-342 (2014).

18. Shafti-Keramat S, Handisurya A, Kriehuber E, Meneguzzi G, Slupetzky K, Kirnbauer R. Different heparan sulfate proteoglycans serve as cellular receptors for human papillomaviruses. J. Virol. 77(24), 13125-13135 (2003).

19. Surviladze Z, Dziduszko A, Ozbun MA. Essential roles for soluble virion-associated heparan sulfonated proteoglycans and growth factors in human papillomavirus infections. PLoS Pathog. 8(2), e1002519 (2012).

20. Uhlén M, Björling E, Agaton C et al. A Human Protein Atlas for normal and cancer tissues based on antibody proteomics. Mol. Cell. Proteomics 4(12), 1920-1932 (2005).

21. Uhlén M, Fagerberg L, Hallström BM et al. Tissue-based map of the human proteome. Science 347(6220), (2015).

22. Harris L, McFarlane-Majeed L, Campos-León K, Roberts S, Parish JL. The cellular DNA helicase ChIR1 regulates chromatin and nuclear matrix attachment of the human papillomavirus $16 \mathrm{E} 2$ protein and high copy-number viral genome establishment. $J$. Virol. 91(1), pii: e01853-16 (2017).

23. Chae YK, Ranganath K, Hammerman PS et al. Inhibition of the fibroblast growth factor receptor (FGFR) pathway: the current landscape and barriers to clinical application. Oncotarget 8(9), 16052-16074 (2017). 
24. Porta R, Borea R, Coelho A et al. FGFR: a promising druggable target in cancer: molecular biology and new drugs. Crit. Rev. Oncol. Hematol. 113, 256-267 (2017).

25. De Luca A, Frezzetti D, Gallo M, Normanno N. FGFR-targeted therapeutics for the treatment of breast cancer. Expert Opin. Investig. Drugs 26(3), 303-311 (2017). 